\title{
DECOMPOSITION OF THE PRODUCT OF CYCLES BASED ON DEGREE PARTITION
}

\author{
Y.M. Borse AND S.R. SHAIKH \\ Department of Mathematics \\ Savitribai Phule Pune University \\ Pune-41100\%, India \\ e-mail: ymborse11@gmail.com \\ shazia_31082@yahoo.co.in
}

\begin{abstract}
The Cartesian product of $n$ cycles is a $2 n$-regular, $2 n$-connected and bipancyclic graph. Let $G$ be the Cartesian product of $n$ even cycles and let $2 n=n_{1}+n_{2}+\cdots+n_{k}$ with $k \geq 2$ and $n_{i} \geq 2$ for each $i$. We prove that if $k=2$, then $G$ can be decomposed into two spanning subgraphs $G_{1}$ and $G_{2}$ such that each $G_{i}$ is $n_{i}$-regular, $n_{i}$-connected, and bipancyclic or nearly bipancyclic. For $k>2$, we establish that if all $n_{i}$ in the partition of $2 n$ are even, then $G$ can be decomposed into $k$ spanning subgraphs $G_{1}, G_{2}, \ldots, G_{k}$ such that each $G_{i}$ is $n_{i}$-regular and $n_{i}$-connected. These results are analogous to the corresponding results for hypercubes.
\end{abstract}

Keywords: hypercube, Cartesian product, $n$-connected, regular, bipancyclic, spanning subgraph.

2010 Mathematics Subject Classification: 05C40, 05C70, 68R10.

\section{REFERENCES}

[1] B. Alspach, J.-C. Bermond and D. Sotteau, Decomposition into cycles I: Hamilton decompositions, in: Cycles and Rays, G. Hahn, G. Sabidussi and R.E. Woodrow $(\mathrm{Ed}(\mathrm{s}))$, (Springer, Dordrecht, 1990) 9-18.

doi:10.1007/978-94-009-0517-7_2

[2] B. Alspach and M. Dean, Honeycomb toroidal graphs are Cayley graphs, Inform. Process. Lett. 109 (2009) 705-708.

doi:10.1016/j.ipl.2009.03.009

[3] Y.M. Borse and S.A. Kandekar, Decomposition of hypercubes into regular connected bipancyclic subgraphs, Discrete Math. Algorithms Appl. 7(3) (2015) Article 1550033. doi:10.1142/S1793830915500330 
[4] Y.M. Borse, A.V. Sonawane and S.R. Shaikh, Connected bipancyclic isomorphic mfactorizations of the Cartesian product of graphs, Australas. J. Combin. 66 (2016) $120-129$.

[5] Y.M. Borse and B.N. Waphare, On removable and non-separating even cycles in graphs, J. Indian Math. Soc. 80 (2013) 221-234.

[6] X.-B. Chen, Panconnectivity and edge-pancyclicity of multidimensional torus networks, Discrete Appl. Math. 178 (2014) 33-45. doi:10.1016/j.dam.2014.06.021

[7] S.I. El-Zanati and C.V. Eynden, Cycle factorizations of cycle products, Discrete Math. 189 (1998) 267-275. doi:10.1016/S0012-365X(98)00053-3

[8] M.F. Foregger, Hamiltonian decompositions of products of cycles, Discrete Math. 24 (1978) 251-260. doi:10.1016/0012-365X(78)90096-1

[9] B. Jackson, Removable cycles in 2-connected graphs of minimum degree at least four, J. Lond. Math. Soc.(2) 21 (1980) 385-392. doi:10.1112/jlms/s2-21.3.385

[10] A. Kotzig, Every Cartesian product of two circuits is decomposable into two Hamiltonian circuits (Centre de Recherches Mathématiques, Montréal, 1973).

[11] F.T. Leighton, Introduction to Parallel Algorithms and Architectures: Arrays, Trees, Hypercubes (Morgan Kaufmann, 1992).

[12] T.-K. Li, C.-H. Tsai, J.J.-M. Tan and L.-H. Hsu, Bipanconnectivity and edge faulttolerant bipancyclicity of hypercubes, Inform. Process. Lett. 87 (2003) 107-110. doi:10.1016/S0020-0190(03)00258-8

[13] W. Mader, Kreuzungsfreie $a, b$-Wege in endlichen Graphen, Abh. Math. Semin. Univ. Hambg. 42 (1974) 187-204. doi:10.1007/BF02993546

[14] W. Mader, Connectivity and edge-connectivity in finite graphs, in: Surveys in Combinatorics, B. Bollobás (Ed(s)), (Cambridge University Press, 1979) 66-95. doi:10.1017/CBO9780511662133.005

[15] A.V. Sonawane and Y.M. Borse, Decomposing hypercubes into regular connected subgraphs, Discrete Math. Algorithms Appl. 8(4) (2016) Article 1650065. doi:10.1142/S1793830916500658

[16] M. Xu, J.-M. Xu, X.-M. Hou, Fault diameter of Cartesian product graphs, Inform. Process. Lett. 93 (2005) 245-248. doi:10.1016/j.ipl.2004.11.005 